\title{
Using the Galerkin method to compute the eigenvalues and eigenelements of the second-order Sturm-Liouville problems
}

\author{
W. Abbas ${ }^{\mathrm{a}}$, O. Mokhtar, ${ }^{\mathrm{b}, *}$, Mohamed Fathy ${ }^{\mathrm{a}}$, Ahmed A. M. Said ${ }^{\mathrm{b}}$,Hesham A. M. A \\ a Basic and Applied Science Department, College of Engineering and Technology, Arab Academy for Science, Technology, and Maritime Transport, Cairo, \\ Egypt.
b Physics and Engineering Mathematics Department, Faculty of Engineering, Mattaria, Helwan University, Egypt.
}

\begin{abstract}
A B S T R A C T
In this paper, an efficient method based on the Galerkin technique for computing the eigenvalues and eigenfunctions for the second-order Sturm-Liouville problems. The first kind of Chebyshev polynomials $T_{n}(x)$ is used as basis functions to solve this problem. The Chebyshev-Galerkin method is applied to reduce an ordinary differential equation into a system of algebraic equations using the orthogonality of Chebyshev polynomials and new relations driven from the orthogonality property. Numerical examples show that the proposed method is an easy method to implement and introduce accurate results.
\end{abstract}

Keywords:

Chebyshev, Galerkin, Eigenvalues, Eigenfunctions, Sturm-Liouville

\section{Introduction}

Sturm-Liouville boundary value problem has played significant roles in many areas of science, engineering, and mathematics. The researchers have studied and many of the associated theories introduced over 170 years ago. The solution of various problems in the fields of mathematics, physics, and engineering is closely related to the solution of Sturm-Liouville problem. In mathematics, some separable partial differential equations have reduced to Sturm-Liouville problem as wave and heat equations that are used for describing vibrations of beams of structures and heat transferred by conduction in rod in addition to many differential equations such as Bessel, Hermite, Jacobi, and Legendre equations can be transformed into SturmLiouville equations as in $[1,2]$. In physics, the solution of Sturm-Liouville problems 
provides a solution of Schrödinger's equation that is a key result in quantum mechanics and illustrates energy state and energy spectrum of systems, for example, hydrogen atoms. The Sturm-Liouville theory has been the basis for the development of fields such as the spectral theory of differential operators, functional space and modern analysis and theory of self-adjoint operators [3].

Many methods have been developed to solve one-dimensional SturmLiouville problems, among these methods, finite difference methods [4, 5]. S. Pruess replaced the coefficients of Sturm-Liouville equation with piecewise polynomials functions to solve it. Recently, Hargrave [7] solved linear Sturm-Liouville eigenvalue problems by reducing the second-order Sturm- Liouville into a system of first-order equations by using Prüfer substitutions. Canosa and Oliveira [8] presented a onedimensional Schrödinger equation that represents the second-order Sturm-Liouville and solved it by using step function. Wittrick and Williams [9] developed an efficient algorithm for computing the natural undamped frequencies (eigenvalues) of vibration of any linearly elastic structure. Jiang et al. [10] studied the existence of second-order singular Sturm-Liouville integral boundary value problems. S. Abbasbandy and A. Shirzadi [11] used the homotopy analysis method for calculating the second and fourthorder Sturm-Liouville. S. Yuan et al. [12] used the exact dynamic stiffens method for solving regular second-fourth-order Sturm-Liouville problem. B. Chanane [13] used a method based on sampling theory to compute the eigenvalues of second-order SturmLiouville problems with parameter-dependent potential and boundary conditions and modified his method to be valid for fourth-order Sturm-Liouville as in [14]. M. I. Syam and H. I. Siyyam $[15,16]$ used variational iteration methods for computing the eigenvalues of fourth and sixth-order Sturm-Liouville problem. B. S. Attili and D. Lesnic[17] used the Adomian decomposition method for getting the eigenelments of fourth-order Sturm-Liouville problem. Based on finite difference and Numerov's methods A. Rattana and C. Böckmann [18] stated their method for computing fourthorder Sturm-Liouville. M. El-Gamel et al. [19] used Galerkin technique and Legendre polynomials as basis functions for computing the eigenelements of fourth-order SturmLiouville.

In this paper, we present a Chebyshev-Galerkin method for solving the second-order Sturm-Liouville boundary value problem in the form:

$$
\frac{d}{d x}\left[p(x) y^{\prime}(x)\right]+q(x) y(x)=\lambda w(x) y(x), \quad-1 \leq x \leq 1,
$$

subject to the boundary conditions

$$
\begin{gathered}
y(-1)=y(1)=0 . \\
\text { ph2 }
\end{gathered}
$$


where $p(x), q(x)$ and $w(x)$ are continuous functions. This equation contains both an unknown function called eigenfunctions $y(x)$ and an unknown parameter called eigenvalues $\lambda$. By using our method, we won't compute the eigenvalues of this problem only, but also we will find eigenfunction at different values $\lambda$.

This article is organized as follows: a brief introduction the preliminaries of Chebyshev polynomials is presented in Section 2. How to use the Chebyshev-Galerkin method to solve the Sturm-Liouville problem introduces in Section 3. The treatment of general solution domain for any problem is introduced in Section 4. Section 5 includes four solved examples of second-order Sturm-Liouville problems. Finally, the conclusions of our results.

\section{Chebyshev polynomials Preliminaries}

In this section, we present some necessary definitions and mathematical preliminaries of Chebyshev polynomials first kind $T_{n}(x)$ and some important properties that are required for our subsequent development. The well-known Chebyshev polynomials are defined on the interval $[-1,1]$ and can be determined at endpoints:

$$
T_{n}(-1)=(-1)^{n}, \quad T_{n}(1)=1 .
$$

The power function $x^{k}$ can be expanded in a Chebyshev series form as:

$$
x^{k}=2^{1-k} \sum_{\substack{j=0 \\ k-j \text { even }}}^{k} \frac{k !}{\left(\frac{k-j}{2}\right) !\left(\frac{k+j}{2}\right) !} T_{j}(x) .
$$

The product between any two Chebyshev polynomials is given by:

$$
2 T_{n}(x) T_{m}(x)=T_{n+m}(x)+T_{|n-m|}(x) .
$$

The orthogonality of polynomials on $[-1,1]$ is given by [20-22]:

$$
\int_{-1}^{1} T_{n}(x) T_{m}(x) \frac{d x}{\sqrt{1-x^{2}}}=\frac{\pi}{2} c_{n} \delta_{n, m}, \forall n, m \geq 0,
$$

where $\delta_{n, m}$ is the Kronecker symbol and 


$$
c_{n}= \begin{cases}2, & \text { if } n=0 \\ 1, & \text { if } n \geq 1, \\ 0, & \text { otherwise }\end{cases}
$$

The $s^{\text {th }}$ derivative of Chebyshev polynomials $T_{n}(x)$ is given as [23] :

$$
\begin{aligned}
T_{n}^{(s)}(x)= & 2^{s} \sum_{j=0}^{\frac{n-s}{2}} n(n-j-1) \underline{s-1} \frac{(s+j-1) !}{j !(s-1) !} T_{n-2 j-s}(x) \\
& -2^{s-1} n[n-s \text { even }]\left(n+\frac{s}{2}-1\right) \frac{s-1}{\left(n+\frac{s}{2}-1\right) !} \frac{(n-1) !\left(n+\frac{s}{2}\right) !}{(x)} T_{0}(x),
\end{aligned}
$$

where falling factorials $(x) \underline{n}=x(x-1) \cdots(x-n+1)$ and inversion symbol $[p]$ which is true 1 if $p$ and 0 otherwise.

The first $T_{n}^{\prime}(x)$ and second derivative $T_{n}{ }^{\prime \prime}(x)$ of Chebyshev polynomial are reduced from above equation.

$$
\begin{aligned}
& T_{n}^{\prime}=2 \sum_{j=0}^{\frac{n-1}{2}} n T_{n-2 j-1}(x)-n[n-1 \text { even }] T_{0}(x) . \\
& T_{n}^{\prime \prime}=4 \sum_{j=0}^{\frac{n-2}{2}} n(n-j-1)(j+1) T_{n-2 j-2}(x)-4[n-2 \text { even }]\left(\frac{n}{2}\right)^{3} T_{0}(x) .
\end{aligned}
$$

The $s^{\text {th }}$ derivative of the polynomials at endpoints is given as [24]:

$$
\left.\frac{d^{s} T_{n}(x)}{d x^{s}}\right|_{x= \pm 1}=( \pm 1)^{n+s} \prod_{k=0}^{s-1} \frac{n^{2}-k^{2}}{2 k+1}, n \geq s
$$

\section{Chebyshev-Galerkin method}

\subsection{Eigenvalues computation}

To compute the eigenvalues of equation (1.1) with boundary conditions (1.2) by using Chebyshev polynomials and Galerkin technique together, we will introduce the 
integration value of the product between $x^{r}, T_{n}(x)$, and $T_{n}^{(s)}(x)$ using the Chebyshev orthogonality.

Lemma 1 For $n, m$ and $r$ are integer values

(i) $\int_{-1}^{1} \frac{x^{r} T_{n}(x) T_{m}(x)}{\sqrt{1-x^{2}}} d x$

$$
=\frac{1}{2^{r}} \sum_{\substack{p=0 \\ r-p \text { even }}}^{r} \frac{r !}{\left(\frac{r-p}{2}\right) !\left(\frac{r+p}{2}\right) !} \frac{\pi}{2} c_{p}\left(\delta_{p, m+n}+\delta_{p,|m-n|}\right) .
$$

(ii) $\int_{-1}^{1} \frac{x^{r} T_{n}^{\prime}(x) T_{m}(x)}{\sqrt{1-x^{2}}} d x$

$$
\begin{array}{r}
2^{1-r} \sum_{j=0}^{\frac{n-1}{2}} \sum_{\substack{p=0 \\
r-p \text { even }}}^{r} n \frac{r !}{\left(\frac{r-p}{2}\right) !\left(\frac{r+p}{2}\right) !} \frac{\pi}{2} c_{p}\left(\delta_{p, n-2 j+m-1}+\delta_{p,|n-2 j-m-1|}\right) \\
-2^{1-r}[n-1 \text { even }] n \sum_{\substack{p=0 \\
r-p \text { even }}}^{r} \frac{r !}{\left(\frac{r-p}{2}\right) !\left(\frac{r+p}{2}\right) !} \frac{\pi}{2} c_{p} \delta_{p, m} .
\end{array}
$$

(iii) $\int_{-1}^{1} \frac{x^{r} T_{n}^{\prime \prime}(x) T_{m}(x)}{\sqrt{1-x^{2}}} d x$

$$
\begin{aligned}
=\frac{2^{2-r} \pi}{2} \sum_{j=0}^{\frac{n-2}{2}} \sum_{\substack{p=0 \\
r-p \text { even }}}^{r} \frac{n(n-j-1)(j+1) r ! c_{p}}{\left(\frac{r-p}{2}\right) !\left(\frac{r+p}{2}\right) !}\left(\delta_{p, n-2 j+m-2}+\delta_{p,|n-2 j-m-2|}\right) \\
\quad-\frac{2^{3-r} \pi}{2}[n-2 \text { even }]\left(\frac{n}{2}\right)^{3} \sum_{\substack{p=0 \\
r-p \text { even }}}^{r} \frac{r !}{\left(\frac{r-p}{2}\right) !\left(\frac{r+p}{2}\right) !} c_{p} \delta_{p, m} .
\end{aligned}
$$

\section{Proof}

(i) By recalling equations (2.2), (2.3) and (2.4), we can write $x^{r}$ in terms of $T_{j}(x)$. By using equation (2.3), the product of $T_{n}(x) T_{m}(x)$ can be as a sum of two Chebyshev polynomials. Finally, by using the orthogonality property (2.4), we can prove (i). To prove lemma (ii) and (iii), $T_{n}^{\prime}(x)$ and $T_{n}^{\prime \prime}(x)$ can be written as $\sum T(x)$ using equation 
(2.6) and (2.7) respectively. Similar to the steps in lemma (i), relations (ii) and (iii) can be proved.

For the sake of simplicity, equation (1.1) can be expanded and written as:

$$
p(x) y^{\prime \prime}(x)+p^{\prime}(x) y^{\prime}(x)+q(x) y(x)=\lambda w(x) y(x),-1 \leq x \leq 1 .
$$

The approximated solution of equation (1.1) in terms of the first kind Chebyshev polynomials $T_{n}(x)$ is given as:

$$
y_{n}(x) \cong \sum_{j=0}^{n} c_{j} T_{j}(x) .
$$

By applying Galerkin method using Chebyshev polynomials as a basis on equation (3.1), yields

$$
\left\langle p(x) y^{\prime \prime}(x), T_{r}(x)\right\rangle+\left\langle p^{\prime}(x) y^{\prime}(x), T_{r}(x)\right\rangle+\left\langle q(x) y(x), T_{r}(x)\right\rangle=\lambda\left\langle w(x) y(x), T_{r}(x)\right\rangle,
$$

where the inner product $\langle.,$.$\rangle is defined as:$

$$
\langle\varphi(x), \psi(x)\rangle=\int_{-1}^{1} \varphi(x) \psi(x) \frac{d x}{\sqrt{1-x^{2}}} .
$$

By replacing each $y(x)$ in the equation (3.3) with the approximate solution defined in (3.2) and expanding the functions $p(x), p^{\prime}(x), q(x)$ and $w(x)$, we introduce the following theorem.

Theorem 3.1 If the assumed approximate solution of (1.1) with boundary condition (1.2) is (3.2), then the discrete Chebyshev-Galerkin system for the determination of the unknown coefficients $\left\{c_{j}\right\}_{j=0}^{n}$ is given by:

$$
\begin{aligned}
\sum_{j=0}^{n} & {\left[\sum_{\kappa_{1}}^{q} a_{\kappa_{1}} \int_{-1}^{1} \frac{x^{\kappa_{1}} T_{j}^{\prime \prime}(x) T_{r}(x)}{\sqrt{1-x^{2}}} d x+\sum_{\kappa_{2}}^{q} a_{\kappa_{2}} \int_{-1}^{1} \frac{x^{\kappa_{2}} T_{j}^{\prime}(x) T_{r}(x)}{\sqrt{1-x^{2}}} d x\right.} \\
& \left.+\sum_{\kappa_{3}=0}^{q} a_{\kappa_{3}} \int_{-1}^{1} \frac{x^{\kappa_{3}} T_{j}(x) T_{r}(x)}{\sqrt{1-x^{2}}} d x\right] c_{j}=\lambda \sum_{j=0}^{n} \sum_{\kappa_{4}=0}^{q}\left[a_{\kappa_{4}} \int_{-1}^{1} \frac{x^{\kappa_{4}} T_{j}(x) T_{r}(x)}{\sqrt{1-x^{2}}} d x\right] c_{j} .
\end{aligned}
$$

The matrix form of the previous theorem is

$$
A \mathbf{c}=\lambda B \mathbf{c},
$$

where 


$$
\begin{aligned}
& A=\left(\begin{array}{l}
S \\
E
\end{array}\right), \quad B=\left(\begin{array}{c}
G \\
O
\end{array}\right), \quad \mathbf{c}=\left(\begin{array}{c}
c_{0} \\
c_{1} \\
\vdots \\
c_{n}
\end{array}\right), \\
& \{S\}_{j, r}=\sum_{\kappa_{1}}^{q} a_{\kappa_{1}} \int_{-1}^{1} \frac{x^{\kappa_{1}} T_{j}^{\prime \prime}(x) T_{r}(x)}{\sqrt{1-x^{2}}} d x \\
& \quad+\sum_{\kappa_{2}}^{q} a_{\kappa_{2}} \int_{-1}^{1} \frac{x^{\kappa_{2}} T_{j}^{\prime}(x) T_{r}(x)}{\sqrt{1-x^{2}}} d x+\sum_{\kappa_{3}}^{q} a_{\kappa_{3}} \int_{-1}^{1} \frac{x^{\kappa_{3}} T_{j}(x) T_{r}(x)}{\sqrt{1-x^{2}}} d x, \\
& \{G\}_{j, r}=\sum_{\kappa_{4}}^{q} a_{\kappa_{4}} \int_{-1}^{1} \frac{x^{\kappa_{4}} T_{j}(x) T_{r}(x)}{\sqrt{1-x^{2}}} d x .
\end{aligned}
$$

and $\{E\}_{j}=\sum_{j=0}^{n} c_{j} T( \pm 1)$ which is deduced from the boundary conditions (1.2) and can be determined from equation (2.1). The terms $\{S\}_{j, r}$ and $\{G\}_{j, r}$ are calculated by using equations Lemma 1. By multiplying equation (3.4) by $B^{-1}$, yields

$$
\phi \mathbf{c}=\lambda \mathbf{c},
$$

where $\phi=B^{-1} A$. Equation (3.5) be in homogenous form as

$$
(\phi-\lambda I) \mathbf{c}=O .
$$

So, the eigenvalues $\lambda$ can be calculated by solving the equation

$$
|\phi-\lambda I|=0 .
$$

\subsection{Eigenfunctions computation}

This section illustrates how eigenfunctions can be calculated. The basic idea of calculations is converting the equation (1.1) into an initial value problem. Assume that $y_{1}(x)$ and $y_{2}(x)$ are the solutions of initial value problem of equation (1.1) by normalizing conditions.

$$
y(-1)=0, \quad y^{\prime}(-1)=1,
$$

and

$$
y(-1)=0, \quad y^{\prime}(-1)=0,
$$


respectively. The eigenfunctions can be obtained:

$$
y_{n}(x)=y_{1}(x)+\beta y_{2}(x),
$$

According to equation (1.2), equation (3.11) can be reformed as:

$$
y_{n}(x)=y_{1}(x)-\frac{y_{1}(1)}{y_{2}(1)} y_{2}(x), \quad \beta=-\frac{y_{1}(1)}{y_{2}(1)} .
$$

\section{Treatment of General solution domain}

The solution domain changes from application to another. In several applications, the solution domain is not necessary to be $[-1,1]$. To solve Sturm-Liouville problem using Chebyshev-Galerkin method discussed in Section 3 with a general solution domain $[a, b]$, we should use the linear transformation:

$$
t=\frac{1}{2}(b-a)(x+1)+a \text {. }
$$

Assume, the general domain of the second-order Sturm-Liouville problem as:

$$
p(t) y^{\prime \prime}(t)+p^{\prime}(t) y^{\prime}(t)+q(t) y(t)=\lambda w(t) y(t), \quad a \leq t \leq b .
$$

By applying the transformation (4.1) into the problem (4.2), yields

$$
\left(\frac{2}{b-a}\right)^{2} p(x) y^{\prime \prime}(x)+\left(\frac{2}{b-a}\right) p^{\prime}(x) y^{\prime}(x)+q(x) y(x)=\lambda w(x) y(x), \quad-1 \leq x \leq 1 .
$$

After solving the last equation by our method, we can get the solution in terms of variable $t$ by using the inverse linear transformation that is used above, we can get the eigenfunctions in terms of $x$.

\section{Numerical results}

In this section, four examples of second-order Sturm-Liouville problems are presented to confirm the accuracy of the proposed method. The eigenvalues and corresponding eigenfunctions for each problem are calculated. The comparison between the results of Chebyshev-Galerkin method and other methods will introduce in this section. All programs carry out by using MATLAB 2012 and Mathematica 10 packages.

Example 5.1 $[25,26]$ Consider the following second-order Sturm-Liouville problem 


$$
y^{\prime \prime}(x)+\lambda y(x)=0, \quad 0 \leq x \leq 1,
$$

with boundary conditions

$$
y(0)=0, \quad y(1)=0 .
$$

The exact solution is given in $[25,26]$

$$
\lambda=(k \pi)^{2}, \quad k=1,2,3, \ldots
$$

The eigenvalues of this problem are displayed in Table 1 beside the exact solution. Fig. 1 shows the eigenfunctions for the first four eigenvalues

Table 1: Comparison of eigenvalues for example 5.1

\begin{tabular}{ccc}
\hline$k$ & $\lambda_{\text {ChebyshevGalerkin }}$ & $\lambda_{\text {Exact }}$ \\
\hline \hline 1 & 9.869604401089358 & 9.869604401089358 \\
2 & 39.47841760435743 & 39.47841760435743 \\
3 & 88.82643960980422 & 88.82643960980422 \\
4 & 157.9136704174297 & 157.9136704174297 \\
5 & 246.7401100272339 & 246.7401100272339 \\
6 & 355.3057584392169 & 355.3057584392169 \\
\hline
\end{tabular}

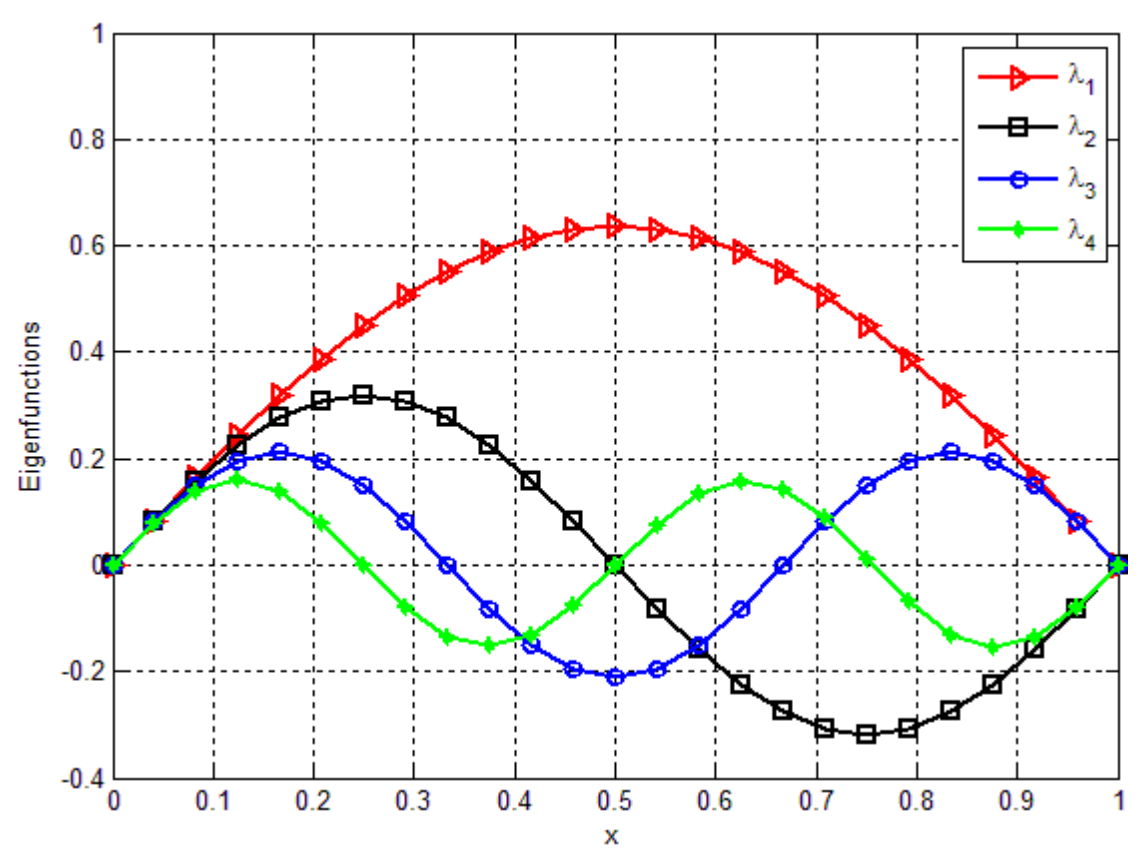

Fig. 1: The first four eigenfunctions for Example 5.1 
Example 5.2 [5, 6] Consider the following second-order Sturm-Liouville problem

$$
x^{6} y^{\prime \prime}(x)+0.75 x^{4} y(x)+\lambda y(x)=0, \quad 1 \leq x \leq 2,
$$

with boundary conditions

$$
y(1)=0, \quad y(2)=0 .
$$

The exact solution is given in $[5,6]$ as $\lambda=\frac{64}{9} k^{2} \pi^{2}, k=1,2,3, \ldots$. The comparison between the eigenvalues of equation (5.2) and exact values are listed in Table 2. The eigenfunctions of the first four eigenvalues are presented in Fig. 2.

Table 2: Comparison of eigenvalues for example 5.2

\begin{tabular}{ccc}
\hline$k$ & $\lambda_{\text {Chebyshev-Galerkin }}$ & $\lambda_{\text {Exact }}$ \\
\hline \hline 1 & 65.646421082678754 & 70.183853518857661 \\
2 & 275.34349367762262 & 280.73541407543064 \\
3 & 625.97865842322415 & 631.65468166971895 \\
4 & 1117.1401432999673 & 1122.9416563017225 \\
5 & 1748.7294360422866 & 1754.5963379714415 \\
6 & 2520.7137863704301 & 2526.6187266788758 \\
\hline
\end{tabular}

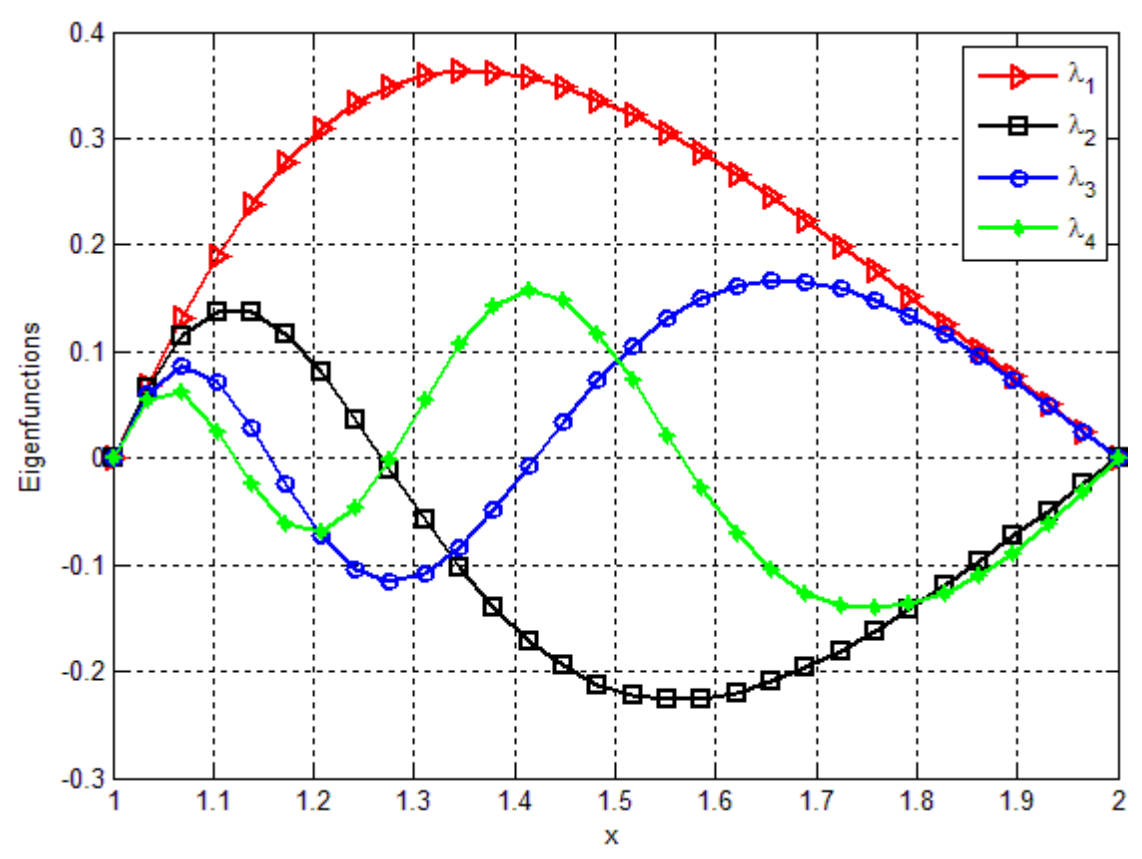

Fig. 2: The first four eigenfunctions for Example 5.2 
Example 5.3 [17] Consider the following second-order Sturm-Liouville problem

$$
y^{\prime \prime}(x)-0.01 x^{2} y(x)+\lambda y(x)=0, \quad 0 \leq x \leq 5,
$$

subject to

$$
y(0)=0, \quad y(5)=0
$$

Equation (5.3) plays a great role in solving the eigenvalues of higher order fourth and sixth order Sturm-Liouville as mention in [27], [17] and [24]. The eigenvalues of fourth-order Sturm-Liouville can be determined by square of the eigenvalues of equation (5.3) and the eigenvalues of sixth-order Sturm-Liouville can be computed by cubic the eigenvalues of equation (5.3). In Table 3, the computed eigenvalues are compared with those obtained by Adomian decomposition method and SLEIGN2 algorithm [29]. Fig. 3 shows the first four eigenfunctions.

Example 5.4 [11] Consider the following second-order Sturm-Liouville problem

$$
x y^{\prime \prime}(x)+y(x)+\lambda x y(x)=0, \quad 0<x<1,
$$

with boundary conditions

$$
y(0)=0, \quad y(1)=0 .
$$

S. Abbasbandy and A. Shirzadi [11] used the homotopy method to solve this problem, while P. Baily et al. [29] used SLEIGN2 algorithm to compute the eigenvalues of above problem. Table 4 shows a comparison between the eigenvalues of equation (5.4) that are computed by our method and the previous methods. Fig. 4 presents the first four eigenfunction.

Table 3: Comparison of eigenvalues for example 5.3

\begin{tabular}{cccc}
\hline$k$ & $\lambda_{\text {Chebysher-Galerkin }}$ & $\lambda_{\text {ADM }}$ & $\lambda_{\text {SLEIGN } 2}$ \\
\hline \hline 1 & 0.46373576996809 & 0.46373576999161 & 0.463735819000 \\
2 & 1.65976201145158 & 1.65976201145916 & 1.659762140000 \\
3 & 3.63529249725374 & 3.63529249724954 & \\
4 & 6.39928275351277 & 6.39928275349180 & \\
5 & 9.95256138202374 & 9.95256138580108 & \\
6 & 14.2953045531725 & 14.2952612249506 & \\
7 & 19.4275685737352 & 19.7523934066243 & \\
\hline
\end{tabular}




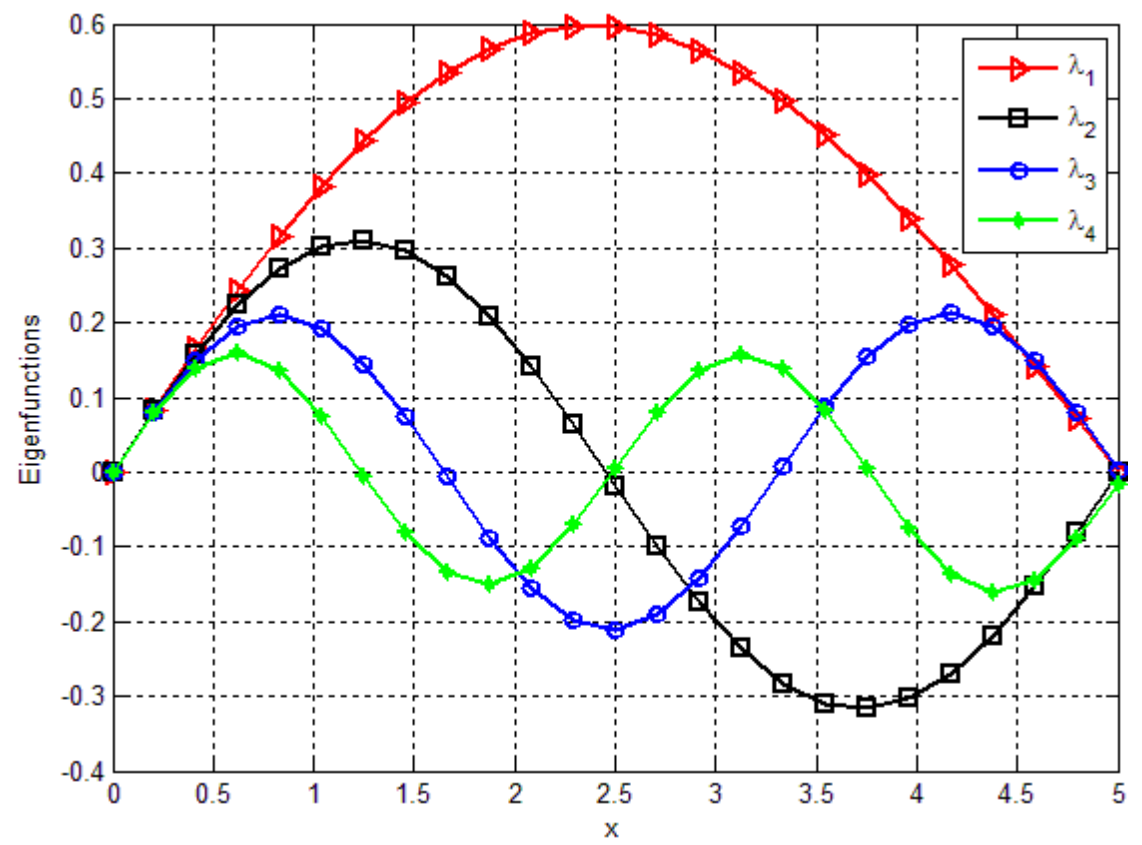

Fig. 3: The first four eigenfunctions for Example 5.3

Table 4: Comparison of eigenvalues for example 5.4

\begin{tabular}{cccc}
\hline$k$ & $\lambda_{\text {Chebyshev-Galerkin }}$ & $\lambda_{\text {Homotopy }}$ & $\lambda_{\text {SLEIGN 2 }}$ \\
\hline \hline 1 & 7.3739850191 & 7.3739850100 & 7.3739900000 \\
2 & 36.336019594 & 36.336018510 & 36.336020000 \\
3 & 85.292582096 & 85.292510750 & 85.292580000 \\
4 & 154.09862374 & 154.10192997 & 154.09862000 \\
\hline
\end{tabular}




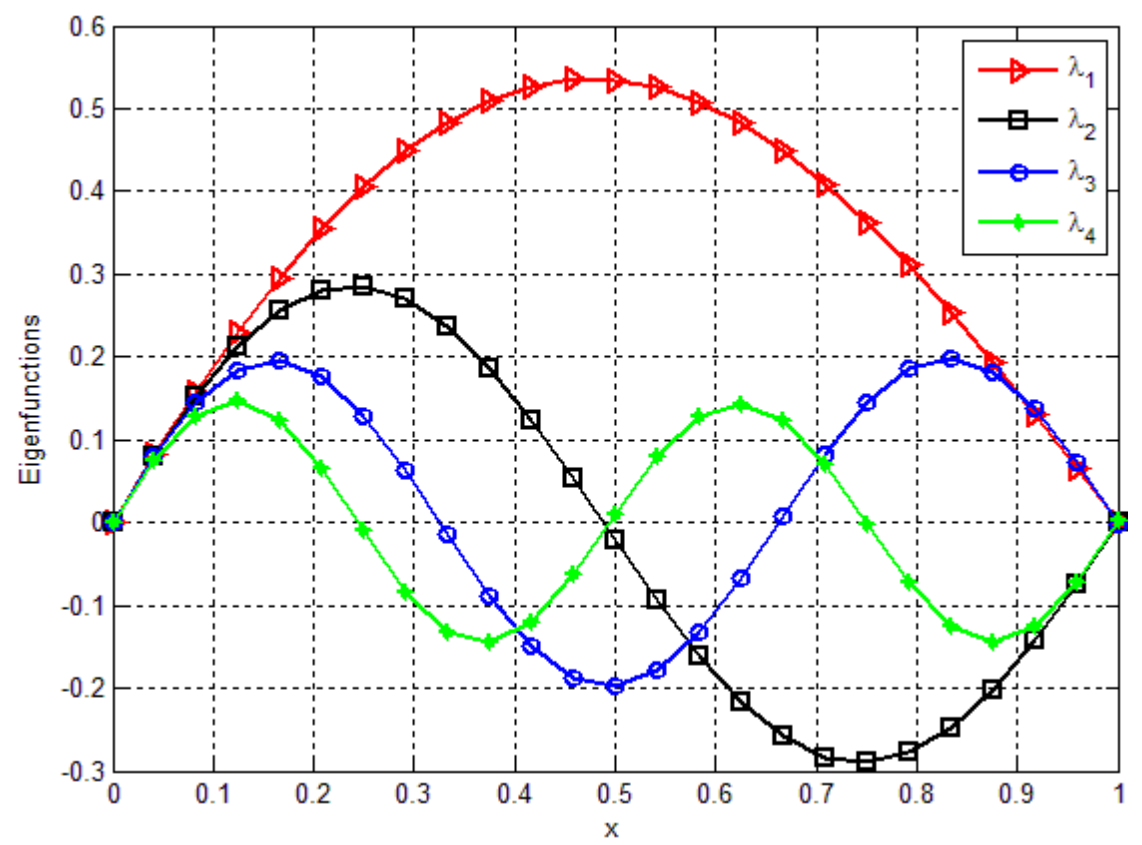

Fig. 4: The first four eigenfunctions for Example 5.4

\section{Conclusion}

In this paper, we introduced an accurate Chebyshev-Galerkin numerical technique for evaluating the eigenvalues and eigenfunctions of second-order Sturm-Liouville boundary value problems. The numerical examples illustrate the efficiency and accuracy of the present method as follows: in Example 1, the eigenvalues are very close to the exact solution. The results are in good agreement which is approaching to the exact solution as in Example 2. The results of Examples 3, 4 are in good agreement with the other methods like Adomian decomposition, homotopy and SLEIGN2 algorithm.

\section{References}

[1] D. G. Zill and W. S. Wright, Advanced Engineering Mathematics. Jones \& Bartlett Learning, 2014.

[2] M. A. Al-Gwaiz, Sturm-Liouville Theory and its Applications. Berlin: Springer, 2008.

[3] G. Freiling and V. Yurko, Inverse Sturm-Liouville Problems and Their Applications. New York: NOVA Science Publishers, 2001.

[4] D. G. Truhlar, "Finite difference boundary value method for solving one-dimensional eigenvalue equations," J. Comput. Phys., vol. 10, no. 1, pp. 123-132, Aug. 1972.

[5] L. Collatz, Eigenwertprobleme und ihre numerische Behandlung. New York: Chelsea, 1948. 
[6] S. Pruess, "Estimating the eigenvalues of Sturm-Liouville problems by approximating the differential equation," J. Numer. Anal., vol. 10, no. 1, pp. 55-68, 1973.

[7] B. A. Hargrave, "Numerical approximation of eigenvalues of Sturm-Liouville systems," J. Comput. Phys., vol. 20, no. 4, pp. 381-396, Apr. 1976.

[8] J. Canosa and R. G. De Oliveira, "A new method for the solution of the Schrödinger equation,” J. Comput. Phys., vol. 5, no. 2, pp. 188-207, Apr. 1970.

[9] W. H. Wittrick and F. W. Williams, "A general algorithm for computing natural frequencies of elastic structures,” Q. J. Mech. Appl. Math., vol. 24, no. 3, pp. 263-284, 1971.

[10] J. Jiang, L. Liu, and Y. Wu, "Second-order nonlinear singular Sturm-Liouville problems with integral boundary conditions," Appl. Math. Comput., vol. 215, no. 4, pp. 1573-1582, Oct. 2009.

[11] S. Abbasbandy and A. Shirzadi, "A new application of the homotopy analysis method: Solving the Sturm-Liouville problems," Commun. Nonlinear Sci. Numer. Simul., vol. 16, no. 1, pp. 112-126, 2011.

[12] S. Yuan, K. Ye, C. Xiao, D. Kennedy, and F. Williams, "Solution of regular second-and fourth-order Sturm-Liouville problems by exact dynamic stiffness method analogy," J. Eng. Math., vol. 86, no. 1, pp. 157-173, 2014.

[13] B. Chanane, "Sturm-Liouville problems with parameter dependent potential and boundary conditions," J. Comput. Appl. Math., vol. 212, no. 2, pp. 282-290, Mar. 2008.

[14] B. Chanane, "Accurate solutions of fourth order Sturm-Liouville problems," J. Comput. Appl. Math., vol. 234, no. 10, pp. 3064-3071, 2010.

[15] M. I. Syam and H. I. Siyyam, "An efficient technique for finding the eigenvalues of fourth-order Sturm-Liouville problems," Chaos Solitons Fractals, vol. 39, no. 2, pp. 659$665,2009$.

[16] H. Siyyam and M. Syam, "An efficient technique for finding the eigenvalues of sixthorder Sturm-Liouville problems," Appl Math Sci, vol. 5, no. 49, pp. 2425-2436, 2011.

[17] B. S. Attili and D. Lesnic, "An efficient method for computing eigenelements of SturmLiouville fourth-order boundary value problems," Appl. Math. Comput., vol. 182, no. 2, pp. 1247-1254, 2006.

[18] A. Rattana and C. Böckmann, "Matrix methods for computing eigenvalues of SturmLiouville problems of order four," J. Comput. Appl. Math., vol. 249, pp. 144-156, 2013.

[19] M. El-Gamel, M. S. El-Azab, and M. Fathy, "An efficient technique for finding the eigenvalues and the eigenelements of fourth-order Sturm-Liouville problems," SeMA J., vol. 74, no. 1, pp. 37-56, 2017.

[20] M. Khader, "Numerical treatment for solving fractional Riccati differential equation," J. Egypt. Math. Soc., vol. 21, no. 1, pp. 32-37, 2013.

[21] J. C. Mason and D. C. Handscomb, Chebyshev Polynomials. Chapman and Hall/CRC, 2002.

[22] J. Shen, "Efficient spectral-Galerkin method II. Direct solvers of second-and fourth-order equations using Chebyshev polynomials," SIAM J. Sci. Comput., vol. 16, no. 1, pp. 7487, 1995.

[23] H. Prodinger, "Representing derivatives of Chebyshev polynomials by Chebyshev polynomials and related questions," Open Math., vol. 15, no. 1, pp. 1156-1160, Sep. 2017. 
[24] Q. M. Al-Mdallal and M. I. Syam, "The Chebyshev collocation-path following method for solving sixth-order Sturm-Liouville problems," Appl. Math. Comput., vol. 232, pp. 391-398, 2014.

[25] R. Johnson, Second-Order Ordinary Differential Equations. Bookboon, 2012.

[26] B. S. Kashkari and M. I. Syam, "An accurate method for solving a class of fractional Sturm-Liouville eigenvalue problems," Results Phys., vol. 9, pp. 560-569, 2018.

[27] D. Lesnic, B. S. Attili, and P. İ. E. B. Metot, "An efficient method for sixth-order SturmLiouville problems," International Journal of Science\&Technology, vol. 2, no. 2, pp. 109-114, 2007.

[28] B. S. Attili and D. Lesnic, "An efficient method for computing eigenelements of SturmLiouville fourth-order boundary value problems," Appl. Math. Comput., vol. 182, no. 2, pp. 1247-1254, 2006.

[29] P. Baily, W. Everitt, and A. Zettl, "Computing Eigenvalues of Singular Sturm-Liouville Problem,” Results Math. vol. 20, pp. 391-423, 1991. 